\title{
La metodología de la investigación en el marco de la agroproducción sustentable
}

\section{The methodology of research in the framework of sustainable agroproduction}

\author{
Manuel E. Milla P. ${ }^{1 *}$ y Danny A. Villegas R. ${ }^{2}$
}

\section{RESUMEN}

El presente trabajo tiene como objetivo presentar una visión acerca de los aspectos metodológicos de la investigación fundamentada en la lógica y la epistemología, con énfasis en la metodología vista como el estudio analítico y crítico de los métodos de investigación y de prueba en el marco de la agroproducción sustentable. Se discute el problema a investigar como punto de iniciación del proceso investigativo, resaltando que la magnitud del problema no es lo más importante, sino el rigor científico como se aborde la alternativa de solución al problema. Además se expone la importancia de diseñar una investigación para garantizar que los resultados obtenidos permitan dar respuesta a los objetivos planteados, por ello se detallan aspectos como población y muestra, así como la necesidad de una ejecución física uniforme de la situación objeto de estudio, así como también se expresa la relevancia del uso correcto de los procedimientos estadísticos en pro de la validez de los procesos de inferencia. Se concluye que las investigaciones deben fortalecer el área del conocimiento y aportar en lo referente a la metodología.

Palabras clave: lógica, epistemología, metodología de la investigación, agroproducción sustentable.

\section{ABSTRACT}

The present work aims to present a vision about the methodological aspects of research based on logic and epistemology, with emphasis on the methodology seen as the analytical and critical study of research and testing methods within the framework of the sustainable agroproduction. The problem to be investigated as a starting point of the investigative process is discussed, highlighting that the magnitude of the problem is not the most important thing, but the scientific rigor as the alternative solution to the problem is addressed. In addition, the importance of designing a research is exposed to guarantee that the obtained results allow to respond to the proposed objectives, for that reason aspects such as population and sample are detailed, as well as the need for a uniform physical execution of the situation under study, as well as as also the relevance of the correct use of statistical procedures in favor of the validity of inference processes is expressed. It is concluded that research should strengthen the area of knowledge and contribute with regard to methodology.

Key words: logic, epistemology, research methodology, sustainable agroproduction.

\footnotetext{
${ }^{1}$ Universidad Nacional Toribio Rodríguez de Mendoza de Amazonas (UNTRM-A), Instituto de Investigación para el Desarrollo Sustentable de Ceja de Selva, Calle Higos Urco N³42-350-356, Calle Universitaria N 304, Chachapoyas, Perú

"Universidad Nacional Experimental de los Llanos Occidentales “Ezequiel Zamora”, Carrera 3 Calle 17, Guanare, Portuguesa, Venezuela.

"Autor de correspondencia. E-mail:manuel.milla@untrm.edu.pe
} 


\section{METODOLOGÍA}

El auge de disciplinas como la lógica, que estudia la estructura, fundamento, y uso de las expresiones del conocimiento humano, y la epistemología, centrada en el estudio crítico del desarrollo, métodos y resultados de las ciencias, conllevan a afirmar que es suficiente una correcta metodología para asegurar el éxito de una investigación. Si bien es cierto que esta afirmación no es exacta, hay que reconocer la importancia que tiene el método en el quehacer científico. Es oportuno aclarar, que el término metodología suele ser usado con dos significados, que tienen en común el estudio del método. En tal sentido, la metodología puede ser vista como una rama de la pedagogía, que se dedica al estudio de los métodos para la transmisión de conocimientos, pero también hay una concepción de la metodología, que la define como el estudio analítico y crítico de los métodos de investigación y de prueba, y en este trabajo se hará énfasis en este enfoque.

Bajo este escenario, podemos conceptualizar la metodología, como la descripción, análisis y valoración crítica de los métodos de investigación. Su propósito fundamental es evaluar los recursos metodológicos, identificar sus limitaciones y analizar ventajas y desventajas de su utilización. Asimismo estamos obligados a establecer diferencias entre los términos, método y técnica, aceptando que el método tiene carácter general y la técnica es particular, pero ambos están orientados a alcanzar los fines de una investigación.

La metodología permite comprender ciertos métodos y técnicas cuya validez ha sido probada en la práctica investigativa, pero bajo ninguna circunstancia nos garantiza el éxito de las mismas, evidentemente nos proporciona los insumos para hacer una valoración crítica de su inserción en el escenario de investigación.

\section{INVESTIGACIÓN}

Una vez revisado lo que es metodología, es conveniente precisar lo que es investigación. Al respecto Einstein señaló que la ciencia consiste en crear teorías: "Ideamos una teoría tras otra, y lo hacemos porque gozamos comprendiendo", esto resalta que el valor esencial de la investigación científica esta soportado en que satisface nuestra curiosidad al ver realizado el deseo de conocer. Al respecto Aristóteles había dicho: "Aprender es el más grande de los placeres no solo para el filósofo, sino también para el resto de la humanidad, por pequeña que sea su capacidad para ello ..." De allí entonces, que la investigación se genera a partir de la identificación de un problema, y nos interesamos en proponer su solución. El exhaustivo proceso para confeccionar la solución, es realmente la investigación.

La investigación nace, cuando detectamos la existencia de un problema, obviamente, hay que contextualizarlo, explorarlo, valorarlo y analizarlo críticamente, para emprender en base a la racionalidad la búsqueda de una probable solución. En tal sentido, la proyección del trabajo de investigación responde a una estructura lógica de decisiones y a una estrategia que garantice la obtención de respuestas adecuadas. La planificación corresponderá entonces a aspectos científicos y administrativos que deben responder al qué y para qué de la investigación, al cuándo y dónde, el cómo y con qué se va a investigar un aspecto de la realidad en el campo de las ciencias naturales o sociales, especialmente en el ámbito de la producción agrícola enmarcada en el paradigma de la sustentabilidad.

\section{EL PROBLEMA A INVESTIGAR}

Muchas personas autocalificadas de investigadores, son de la idea, que cuanto más ambicioso seamos con el problema a investigar mayor será el impacto causado, esto es un asunto de índole paradigmático, ya que si no se delimita adecuadamente el problema, corremos el riesgo de no aceptar que hemos concluido la investigación.

Abordando lo que son los paradigmas y su incidencia en las investigaciones, podemos acotar que en la mayoría de las organizaciones, sin importar su tamaño y naturaleza, las nuevas ideas generan cambios e incertidumbre, motivado precisamente, a que hay una evidente resistencia a los cambios o nuevas concepciones. 
Los paradigmas son modelos, imágenes, supuestos e historias que llevamos en la mente acerca de nosotros, de los demás, de las instituciones y de todos los aspectos del mundo; los paradigmas no son los hechos o las experiencias mismas, solamente nos explican por qué las percibimos o experimentamos de la forma que lo hacemos. En este orden de ideas, los paradigmas establecen límites, pero también proporcionan guías para el éxito.

El no reconocer la necesidad de delimitar el problema, puede ser debido a una parálisis paradigmática, que impide ver que el impacto de una investigación no lo garantiza el tamaño del problema, sino que el problema responda a una necesidad de la sociedad y que sea abordado con el rigor científico que el caso amerite, lo que se traduce en la novedad científica. Esta interpretación de la necesidad de que el problema responda a una necesidad de la sociedad subyace en el hecho de que la ciencia al servicio de la sociedad se traduce en tecnología, y en el escenario de una generación ávida de un desarrollo que se enmarque en el marco de la sustentabilidad se requiere de investigación con pertinencia social pero que persiga fines concretos, en donde se garantice fundamentalmente un equilibrio entre metodología y técnica, puesto que el método científico es el producto de una metodología usada correctamente, una actitud crítica y un intenso trabajo que lleva de la incertidumbre a la probable certeza.

\section{DISEÑO DE INVESTIGACIÓN}

Esto indica que se requiere diseñar la investigación, es decir, trazar la estrategia o secuencia de decisiones de cómo recoger, ordenar, analizar e interpretar los datos provenientes de una investigación, aspecto que exige tener claro cómo se clasifican las investigaciones, según el ambiente en que se realizan, estudios de campo o de laboratorios; según la dirección en que se estudian las variables, estudios prospectivos, correlacionales o ex post facto; y según el origen de los datos, investigaciones fácticas o documentales. Otra clasificación es la que divide la investigación en cuantitativa y cualitativa, además de la expuesta por Campbell y
Stanley (1963), que categoriza las investigaciones en experimentales, cuasiexperimentales y no experimentales, diferenciando esta última en diseños transversales y longitudinales, y sus distintas modalidades.

\section{Población y muestra}

Por razones de tiempo y costos, en reiteradas ocasiones el investigador se ve obligado a procesar información proveniente de un subconjunto de la población llamado muestra, la cual debe ser representativa. Con objeto de orientarnos hacia la representatividad, hay que considerar fundamentalmente, el tamaño y el procedimiento de selección, de forma tal que todos los sectores de la población estén debidamente representados en la muestra, es decir, considerando la calidad y la cantidad. En ese sentido, en los muestreos agrícolas, en donde el investigador se enfrenta a un escenario complejo, más que el tamaño de la muestra, el tipo de muestreo a considerar es el factor determinante a la hora de estudiar una porción de la realidad que refleje las complejidades que plantea la inferencia de parámetros que permitan estudiar la sustentabilidad de los diferentes sistemas de producción en donde intervienen factores sociales, económicos, institucionales, entre otros.

\section{Ejecución física}

Las unidades de investigación o unidades experimentales deben ser manejadas en forma similar, esto con el propósito de garantizar que si se observan diferencias entre ellas, las mismas obedezcan, a las técnicas o procedimientos que están siendo probados y no a descuidos en la ejecución de la investigación, lo cual conduce a la formulación de conclusiones que no se correspondan con la verdad.

\section{ANÁLISIS ESTADÍSTICO}

En primer lugar, es conveniente referirnos a las escalas de medición de las variables, esto es, nominal y ordinal, en el caso cualitativo, e intervalo y proporción, en el caso cuantitativo. Las pruebas estadísticas clásicas, requieren para su aplicación válida, que la variable respuesta sea cuantitativa, además del cumplimiento de ciertas características poblacionales o parámetros, 
como son distribución normal y homogeneidad de varianzas, entre otros, de allí que estas pruebas de denominen paramétricas. Sin embargo, en muchos trabajos de investigación, estos requerimientos no son verificados y en otros casos, no se cumplen, y aún así se observa la aplicación de estas pruebas, hecho que no es más que un uso indiscriminado de las referidas pruebas, lo cual inválida las conclusiones emanadas de estos resultados.

Con el objetivo de garantizar la validez de los análisis estadísticos, en caso de que la variable respuesta siendo cuantitativa incumpla con las características poblacionales antes referidas, o si la variable respuesta es cualitativa, se deben usar pruebas estadísticas cuya aplicación válida no esté sujeta al cumplimiento de ciertas características poblacionales o parámetros, estas pruebas se llaman no paramétricas.

Otro aspecto de relevancia en el análisis estadístico, es reconocer cuando estamos en presencia de muestras independientes o muestras relacionadas, además de diferenciar, casos con más de una variable independiente y una variable respuesta, llamados múltiples, y casos con más de un a variable independiente y más de una respuesta, llamados multivariados.

Esto demuestra que la esencia del análisis estadístico no está en el procesamiento de la información, por el contrario está en la concepción y planificación del experimento de forma tal, que permita obtener la información necesaria para dar respuesta a los objetivos de la investigación. Es así como, la sustentabilidad como resultado de la interacción de diferentes factores sugieren rigurosidad en el proceso de la recolección de los datos que permitan describir los diferentes sistemas de producción agrícola, y en ese sentido, la medida es el arma esencial de la investigación científica, porque sólo a través de ella es cómo podemos calibrar los fenómenos y sus relaciones.

\section{CONCLUSIÓN}

Es conveniente aceptar que las investigaciones deben constituir aportes en dos direcciones, en el área del conocimiento y en el aspecto metodológico, y esto requiere de una rigurosa delimitación del problema, sin temer a la simplicidad del mismo, sino más bien atender al rigor científico como se realizará el abordaje.

Por lo antes expuesto, se puede afirmar que si bien la metodología no es una condición suficiente para garantizar el éxito de una investigación, sin duda alguna, es una condición necesaria. Finalmente, estudiar la sustentabilidad de los diferentes sistemas de producción agrícola está condicionado por aspectos científicos y administrativos que guardan relación directa con el método científico, entendiendo que la búsqueda de soluciones frente a las complejidades que plantea el medio rural requiere de un equilibrio entre metodología y técnica.

\section{REFERENCIAS BIBLIOGRÁFICAS}

Campbell, D. y J. Stanley. 1963. Experimental and Quasi-Experiments Designs for Research on Teaching. Chicago (EEUU): Rand McNall \& Company.

Einstein, A. 1950. "On the generalized theory of gravitation”. Scientific American 182 (4): 1317. 